\title{
Die Klimaeignung für die Landwirtschaft - Methode und Probleme einer gesamtschweizerischen Grundlagenkartierung (mit Kartenbeilagen)
}

\section{Problemstellung}

Die Raumplanung auf allen Ebenen erfordert wissenschaftliche Grundlagen zur Erfassung und Beurteilung der natuirlichen Gegebenheiten. Dabei müssen die einzelnen Teilaspekte durch Spezialisten erfasst und für die Anwendung durch die Raumplanung bereitgestellt werden.

Bei der vorliegenden Studie - einem Projekt des Delegierten für Raumplanung, Eidgenössisches Justiz- und Polizeidepartement - ging es darum, die $\mathrm{Kl}$ imaeignung für die Landwirtschaft in der Schweiz zu untersuchen und zu kartieren (siehe auch LEUZINGER 1978). Bestehende Arbeiten wie diejenige von MAEDER (1970) waren dabei zu berücksichtigen. Detaillierte Untersuchungen über die Klimaeignung für die Landwirtschaft lagen nur für einzelne Gebiete der Schweiz vor (zum Beispiel diejenigen von HAEBERLI 1971 und PRIMAULT 1972a im Kanton Waadt).

Ein Agronom (Herr Philippe VAUTIER, ing.agr.ETH, Eidgenössische landwirtschaftliche Forschungsanstalt Changins bei Nyon) und ein Geograph (der Autor, Geographisches Institut der Universität Bern, Abteilung Professor B. MESSERLI) teilten sich in dieser interdisziplinären Arbeit. Eine ausführliche Beschreibung der Methodik und der wissenschaftlichen Unterlagen findet sich bei JEANNERET + VAUTIER 1977a, die Karten sowie die zugehörigen Erläuterungen bei JEANNERET + VAUTIER 1977b.

2. Beziehungen zwischen $\mathrm{Kl}$ ima und landwirtschaftlichen Erträgen

Dass eine Abhängigkeit zwischen Klima und Wetter einerseits und landwirtschaftlichen Erträgen anderseits bestehen muss, liegt auf der Hand. Diese Beziehung quantitativ zu fassen ist jedoch sehr schwierig. MAEDER (1970) und andere beziehen sich vor allem auf jährliche und jahreszeitliche Mittelwerte der $\mathrm{kl}$ imatologischen Messungen, wogegen sich PRIMAULT $(1968,1972 b)$ auf Häufigkeitsanalysen stützt (Quartilmethode).

Bei der vorliegenden Untersuchung wurden zunächs Wege gesucht, um präzisere Beziehungen zwischen Klima und Erträgen zu finden. Diese lassen sich jedoch erst dann bestimmen, wenn die Erträge dem Ablauf der Witterung eines einzelnen Jahres ge- genuiber gestellt werden. Das heisst, dass eine Korrelation zwischen Witterung und Ertrag nur für einzelne Jahre, nicht aber für das Mittel mehrerer Jahre gefunden werden kann.

Für die Untersuchung der Klimaeignung für die Landwirtschaft in der Schweiz wurden die Beziehungen zwischen Erträgen von Futterbau, Getreide und Kartoffeln hauptsächlich aufgrund der Periode 1960 bis 1972 erarbeitet. Die kantonalen landwirtschaftlichen Schulen sowie die eidgenössischen Verwaltungen und Forschungsanstalten lieferten Daten über die landwirtschaftlichen Erträge.

\begin{tabular}{|c|c|c|c|c|c|}
\hline \multicolumn{3}{|c|}{ MUNATSPERIUDEN } & \multicolumn{3}{|c|}{ MEHRMONATIGE PERIODEN } \\
\hline Periode & $\begin{array}{l}\text { Schwelle } \\
\text { unterer }\end{array}$ & $\begin{array}{l}\text { enwerte } \\
\text { loberer }\end{array}$ & Periode & $\begin{array}{l}\text { Schwelle } \\
\text { unterer }\end{array}$ & $\begin{array}{l}\text { enwerte } \\
\text { loberer }\end{array}$ \\
\hline Sept. & 30 & 150 & $\begin{array}{l}\text { Sept. } \\
\text { - Nov. }\end{array}$ & 100 & 400 \\
\hline okt. & 30 & 150 & $\begin{array}{l}\text { Dez. } \\
\text { - Febr. }\end{array}$ & 100 & 450 \\
\hline Nov. & 30 & 150 & $\begin{array}{l}\text { März } \\
- \text { Mai }\end{array}$ & 140 & 350 \\
\hline Dez. & 20 & 250 & $\begin{array}{l}\text { Juni } \\
\text { - Aug. }\end{array}$ & 120 & 400 \\
\hline Jan. & 20 & 250 & $\begin{array}{l}\text { April } \\
- \text { Mai }\end{array}$ & 80 & 250 \\
\hline Febr. & 20 & 150 & $\begin{array}{l}\text { Mai } \\
\text { - Juni }\end{array}$ & 120 & 250 \\
\hline März & 30 & 150 & $\begin{array}{l}\text { Juni } \\
\text { - Juli }\end{array}$ & 90 & 250 \\
\hline April & 30 & 150 & $\begin{array}{l}\text { März } \\
\text { - Aug. }\end{array}$ & 300 & 700 \\
\hline $\operatorname{Mai}$ & 40 & 150 & $\begin{array}{l}\text { Mar } \\
\text { - Aug. }\end{array}$ & 200 & 550 \\
\hline Juni & 40 & 150 & $\begin{array}{l}\text { April } \\
- \text { Ukt. }\end{array}$ & 350 & 800 \\
\hline Juli & 30 & 150 & & & \\
\hline Aug. & 30 & 150 & & & \\
\hline
\end{tabular}

Tabelle 1 :

System von Perioden und Schwellenwerten für das landwirtschaftliche Jahr (September bis August). Niederschlagsmengen ( in mm pro Monat) für Getreide.

François Jeanneret, Dr. phil. nat., Seminarlehrer, Schulhausstr. 19c, 3114 Oberwichtrach. 
Die Beziehungen wurden in Form eines Systems von agroklimatischen Schwellenwerten festgehalten. Für jede Kultur wurde das Jahr in eine bestimmte Zahl von ein- oder mehrmonatigen Perioden aufgeteilt. Jeder Periode wurde ein oberer und ein unterer Schwellenwert für verschiedene Kulturen zugeordnet.

Die Tabelle 1 zeigt als Beispiel Perioden und Schwellenwerte der Niederschlagsmengen für Getreide. Liegt ein Beobachtungswert für eine Periode ausserhalb der zugehörigen Schwellenwerte, so wird der Ertrag im betreffenden Jahr beeinträchtigt. Aus der Häufigkeit solchermassen "gestörter" Perioden lässt sich - unter Berücksichtigung weiterer Komponenten - auf die Ertragsmöglichkeiten für das betreffende Jahr schliessen.

\section{Die klimatologischen Grundlagen}

Die wichtigste Quelle für klimatologisches Beobachtungsmaterial in der Schweiz ist die Meteorologische Zentralanstalt (MZA). Aus technischen Gründen musste für die vorliegende Untersuchung die Auswahl des Datenmaterials auf bereits abgelochte Monatsmittel beschränkt werden. Niederschlagsmengen standen für 307 Stationen, Niederschlagstage (mit mehr als $1 \mathrm{~mm}$ lliederschlag) für 274 Stationen und die Temperaturmittel für 91 Stationen zur Verfügung, im allgemeinen für die Zeit von 1901 bis 1970 oder 1972. Neben diesem umfangreichen Material über eine halbe Million Monatswerte - fanden auch zahlreiche Arbeiten auf nationaler, regionaler und lokaler Ebene Verwendung. Alle neueren $\mathrm{klimatologischen} \mathrm{und} \mathrm{agroklimatischen} \mathrm{Un-}$ terlagen der Schweiz wurden gesichtet und in einer $\mathrm{kl}$ imatologischen Bibliographie der Schweiz für die Jahre 1921 bis 1973 aufgefuihrt. (JEANNERET 1975a). Als wesentlichste klimatoloaische Grundlagen auf nationaler Ebene sind die "Klimatologie der Schweiz" (SCHWEIZ.METEOROLOGISCHE ZENTRALANSTALT, ab 1959), die Karten im "Atlas der Schweiz" (IMHOF, ab 1965) und die Niederschlagskarte von UTTINGER (1949) zu erwähnen.

Im Rahmen der vorliegenden Untersuchung wurden als Feldaufnahme die Spätfrostschäden des Frühjahrs 1974 kartiert (JEANNERET 1975b). Obwohl diese einmalige Aufnahme nicht eine allgemein gültige Frostgefährdung widerspiegelt, lässt sie doch auf Gesetzmässigkeiten der räumlichen Verteilung der Frostschäden schliessen.

\section{Die Bestimmung der Klimaeignung}

Aufgrund des Beobachtungsmateriales der MZA wurde für jede Station und landwirtschaftliche Hauptkultur (Futterbau, Getreide und Kartoffeln) die Häufigkeit guter, mittlerer und schlechter Jahre festgelegt. Die Ergebnisse dieser durch einen Computer durchgeführten Untersuchung bildeten die Grundlage für die Bestimmung - unter Berijcksichtigung weiterer Gesichtspunkte - der Klimaeignung der einzelnen Stationen für die Hauptkulturen.
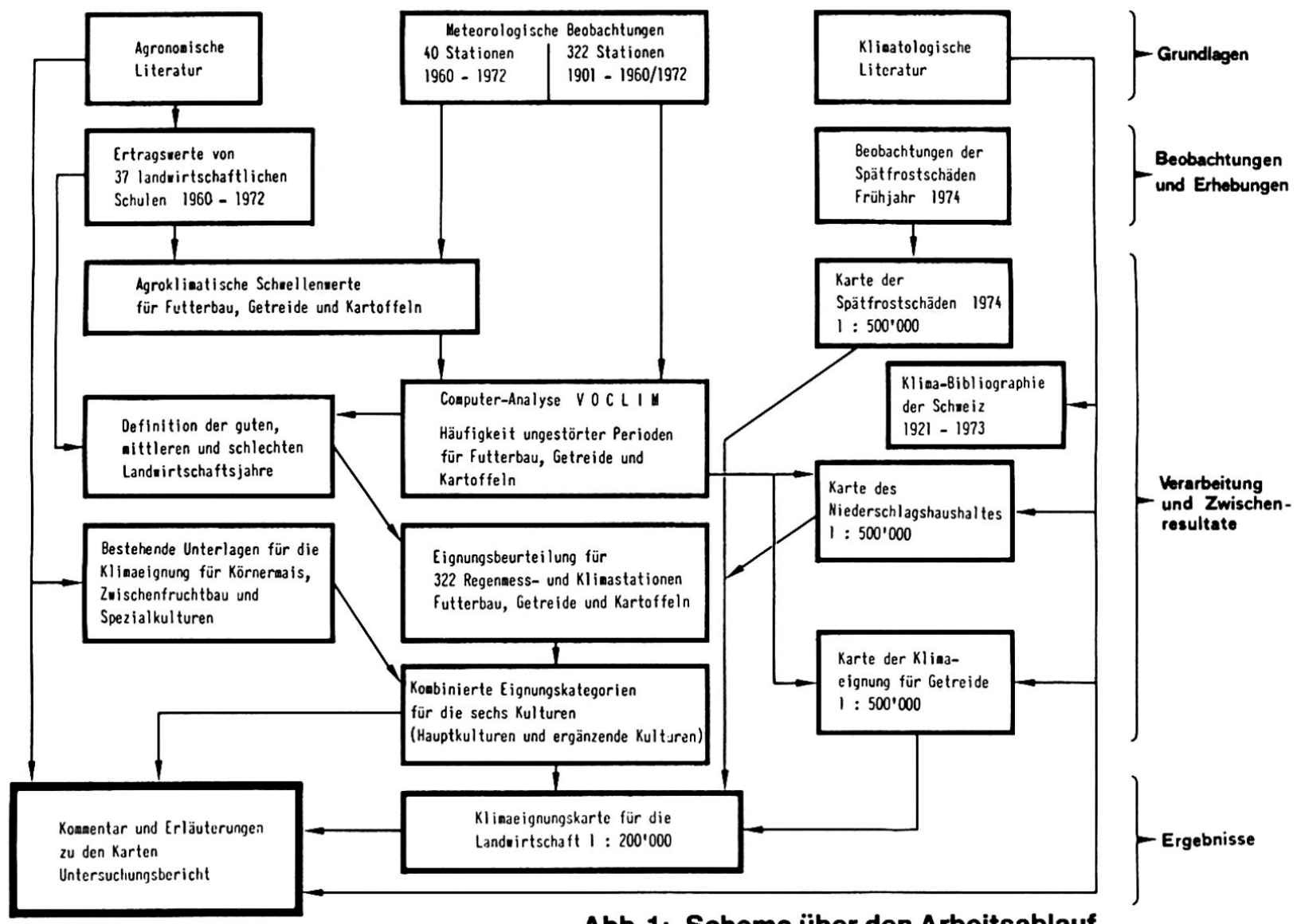

Abb. 1: Schema über den Arbeitsablauf 
Ferner wurde die Klimaeignung für drei weitere Kulturen aus bestehenden Unterlagen entnommen: Körnermais (hauptsächlich nach PRIMAULT 1972a), Zwischenfruchtbau und Spezialkulturen (hauptsächlich nach SCHREIBER et alii 1977). Die Abbildung 1 vermittelt einen schematischen Ueberblick über den gesamten Vorgang.

\section{Die Klimaeignungskategorien}

Die Kombination der Klimaeignung für die sechs Kulturen stellte die Grundlage für die Bestimmung der Eignungskategorien dar. Aus allen möglichen Kombinationen wurden total zwanzig Klassen gebildet, die nachfolgend als "Zonen" bezeichnet werden. Jede der 322 Stationen konnte einer dieser Zonen zugeordnet werden. Eine kurze Beschreibung der Zonen findet sich auf der beiliegenden Legende, während die wichtigsten Kriterien auf der Ruickseite der Klimaeignungskarten (Beilage zu JEANNERET + VAUTIER 1977b) aufgefüht sind.

Aus den klimatischen Eigenschaften der verschiedenen Zonen ergab sich eine Systematik in Abhängigkeit von zwei Faktorengruppen. Einerseits spielen die Dauer der Vegetationsperiode und damit auch die thermischen Verhältnisse (in Abhängigkeit der Höhenlage u.a.) eine grosse Rolle. Anderseits ist der für die Vegetation wirksame Niederschlagshaushalt von Bedeutung, der Frequenz und Ergiebigkeit der Niederschläge in Abhängigkeit des Pflanzenwachstums umfasst.

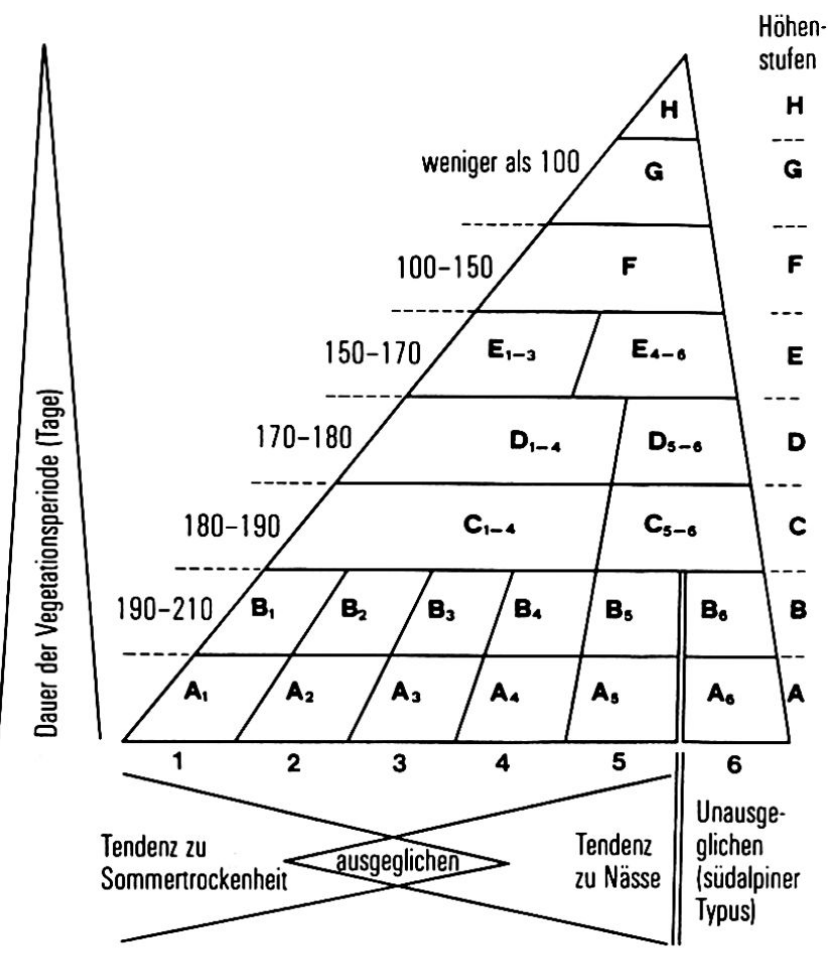

Niederschlagshaushalt

Abb.2: Verteilungsschema der Eignungszonen (Dreiecksflächen $A_{1}$ bis $H$ ) in Abhängigkeit der Vegetationsperiode (Ordinate $A$ bis $H$, nach SCHREIBER et alii 1977) und des Niederschlagshaushaltes (Abszisse 1 bis 6).
In dieser Systematik von Vegetationsdauer und Niederschlagshaushalt sind die Zonen so verteilt, dass höhere Lagen im Gebirge ohnehin feuchter sind und die Differenzierung in verschiedene Typen des Niederschlagshaushaltes weniger sinnvoll wird. Das Dreieck in Abbildung 2 ist aus diesem Grund nicht symmetrisch.

\section{Die Kartierung der $\mathrm{Kl}$ imaeignung}

Für die Praxis genügt die Bestimmung der $\mathrm{Kl}$ imaeignung für einzelne Punkte (Beobachtungsstationen) nicht. Verlangt wird eine flächenhafte Darstellung, also eine Kartierung. Im vorliegenden Fall wurde die Klimaeignung im Massstab 1:200'000 für die ganze Schweiz kartiert (siehe beiliegendes Kartenmuster).

Die Extrapolation von punkthaften Informationen stellt heikle Probleme, geht es doch darum, möglichst alle Faktoren zu berücksichtigen.Es standen für die Schweiz $322 \mathrm{Klima-}$ und Regenmessstationen zur Verfügung, das hejsst 1 Station auf durchschnittlich etwa $130 \mathrm{~km}^{2}$.

Ein besonderes Problem stellt die Kartierung von Gebieten dar, in welchen keine Station liegt. Die Festlegung der Grenzen zwischen einzelnen Zonen erfolgte in erster Linie aufgrund der Höhenverhältnisse, aber auch unter Berücksichtigung anderer Faktoren. Sehr oft wurden diese Grenzen auf funktionale Geländescheiden verlegt, wie Steilstufen, Wälder, Flussläufe, Siedlungen. Diese Landschaftseinheiten sind oftmals sehr wirksame geländekl imatische Grenzen.

Nach IMHOF (1972) ist es nicht sinnvoll, an Hanglagen die Abfolge sämtlicher Stufen darzustellen, da die einzelnen Flächen meistens keine praktische Bedeutung erlangen.

(siehe Landschaftsprofile, Abb.3).

Gesondert wurde die Klimaeignung einer einzelnen Kultur kartiert. Die Klimaeignungskarte für Getreide im Massstab 1:500'000 zeigt die Anbaumöglichkeiten für die wichtigste Kultur. Im selben Massstab entstand auch eine Karte des für den Pflanzenwuchs wirksamen Niederschlagshaushaltes. Diese beiden Karten wie auch die vier Blätter der Klimaeignungskarte für die Landwirtschaft im Massstab 1:200'000 wurden als Beilagen zu JEANNERET + VAUTIER (1977b) publiziert.

\section{Diskussion einiger Ergebnisse}

Die Klimaeignungskarten für die Landwirtschaft vermitteln ein synthetisches Bild des klimabedingten Anbaupotentials im Gebiet der Schweiz. Sie widerspiegeln im wesentlichen die Möglichkeiten, die die traditionelle Landwirtschaft seit langer Zeit wahrnimmt. So erscheint das tiefere Waadtland als günstiges Getreidegebiet, das für Grasbau eher zu trocken ist, das Walliser Rhonetal als für Spezialkulturen (Obst-, Gemüse- und Rebbau) geeignet und das Glarnerland als ein bevorzugtes Viehzuchtgebiet. Es lässt sich aber auch zeigen, dass der unausgeglichene 


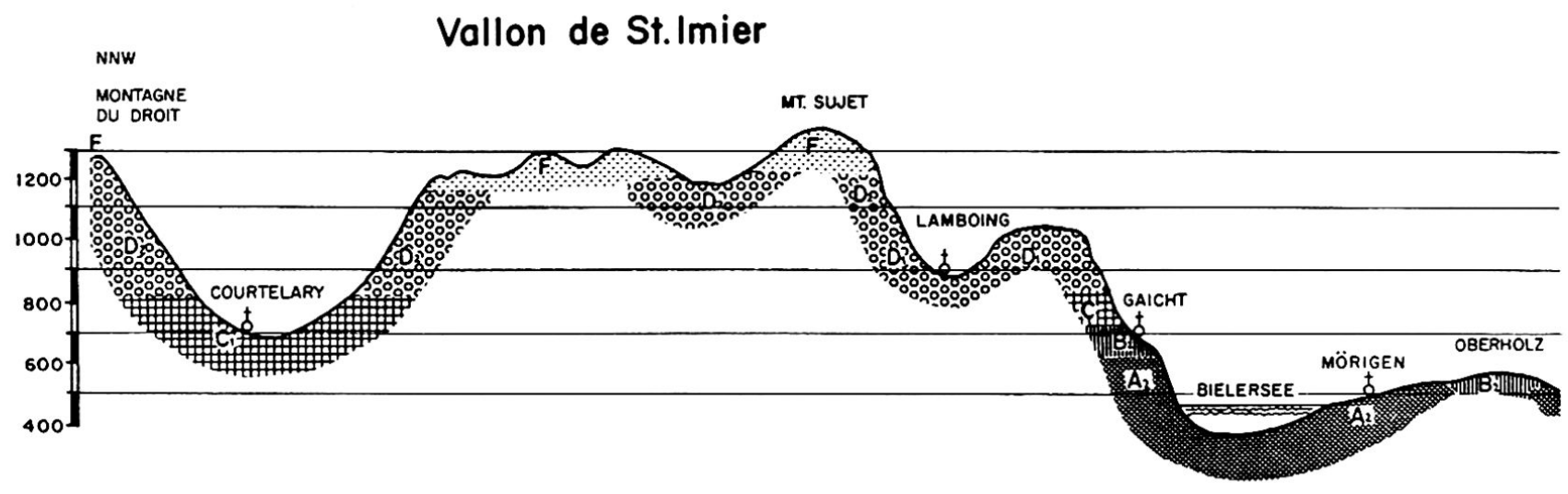

Längenberg - Gürbe - und Aaretal

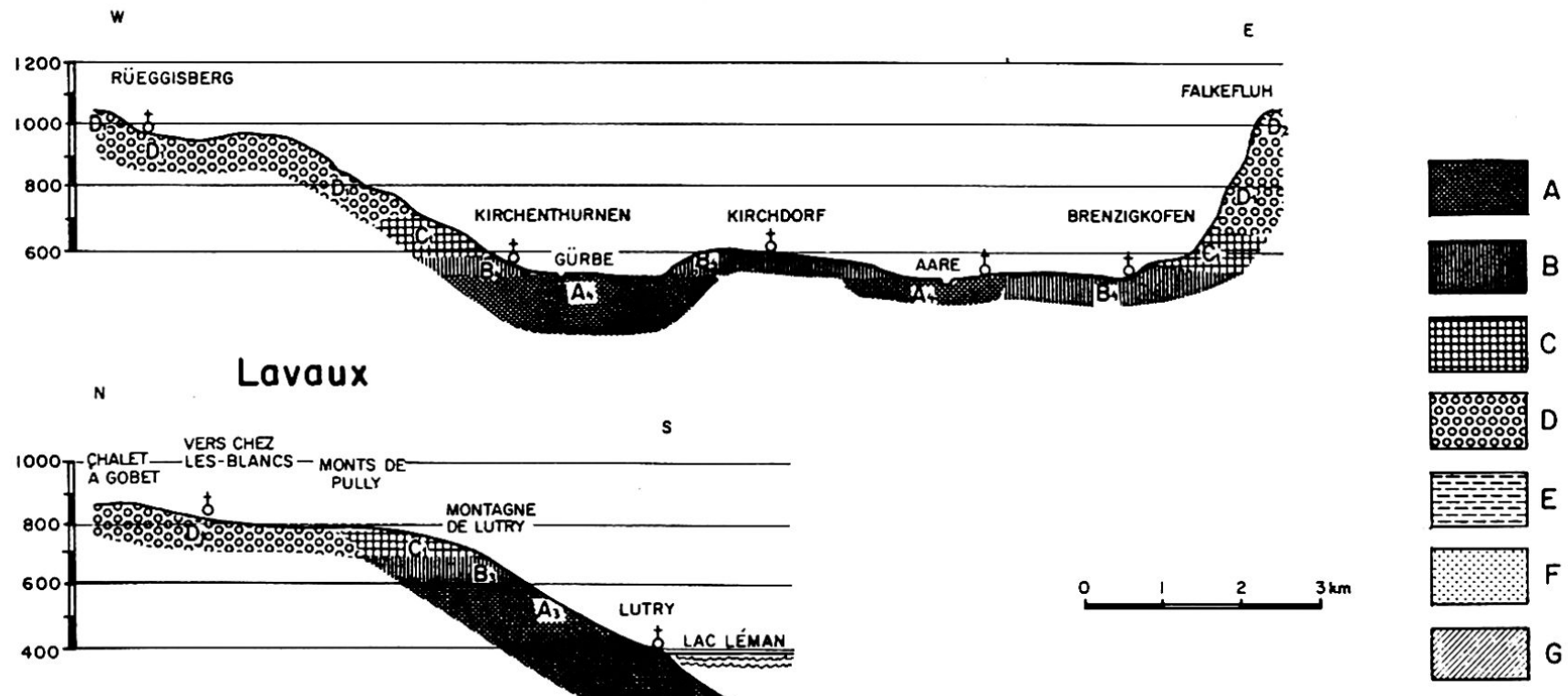

\section{Linth-Tal bei Glarus}

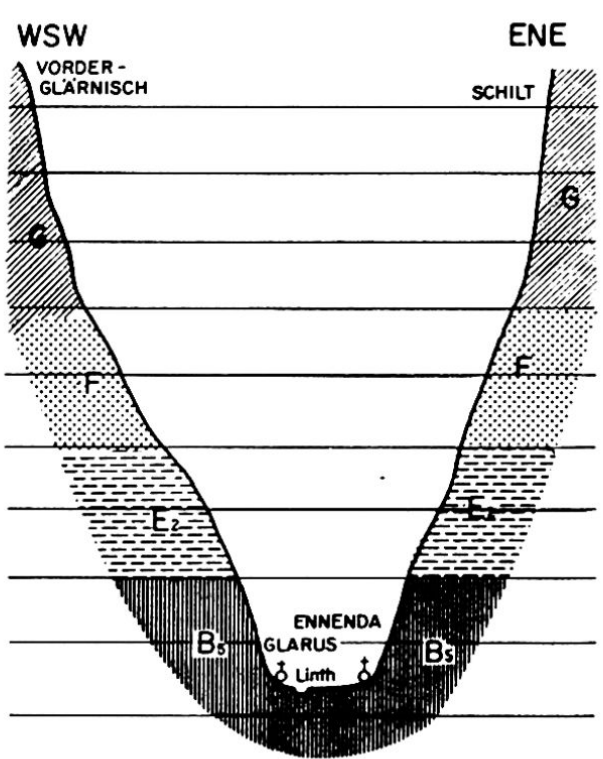

Rheintal bei Chur

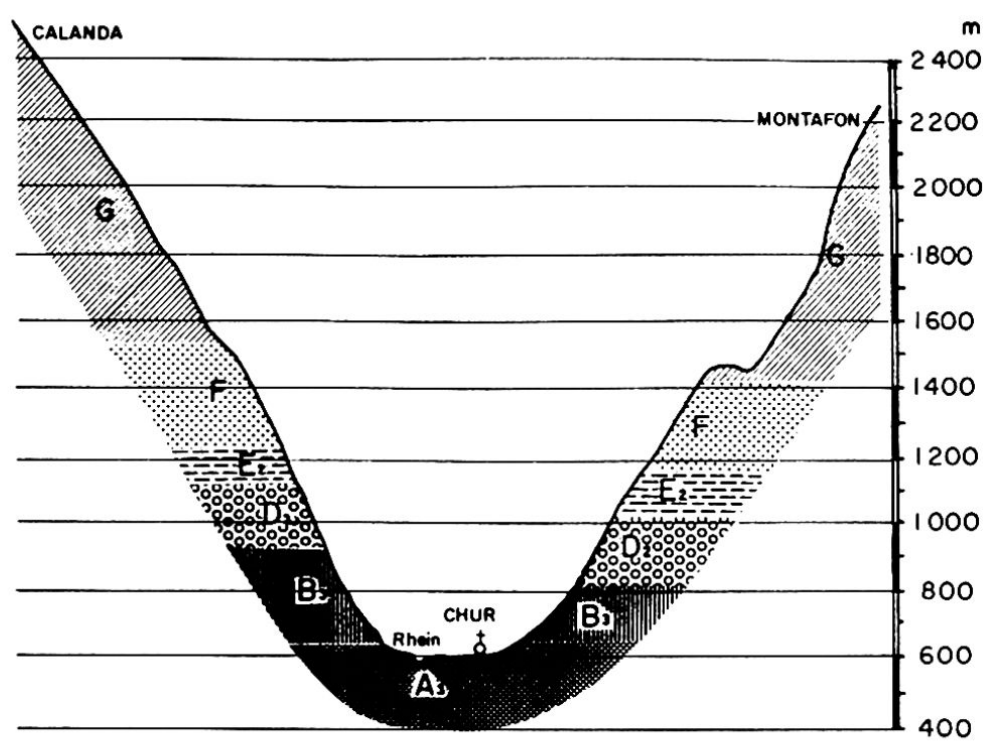

Abb. 3: Profile der Klimaeignung für die Landwirtschaft durch ausgewählte Landschaften des Juras, des Mittellandes und der Alpen. 
C5.6.

83

\%
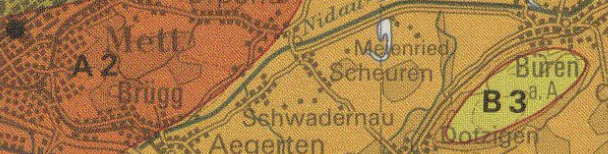

$\mathrm{B}^{\mathrm{a}}$

B 3

3

A 3

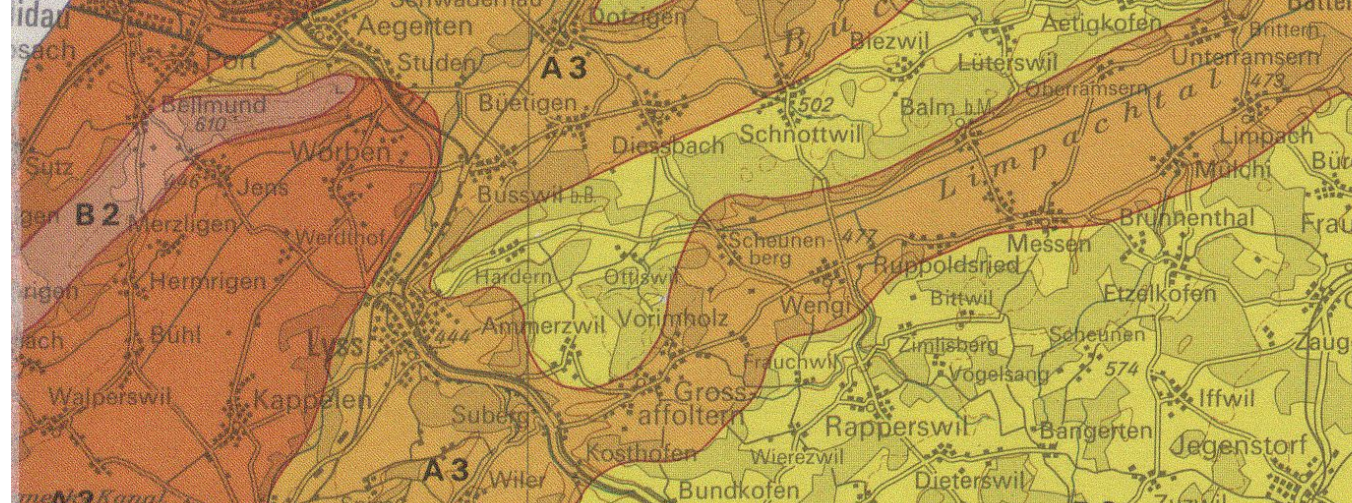

$-x_{2} 2$

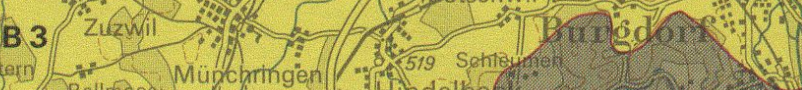
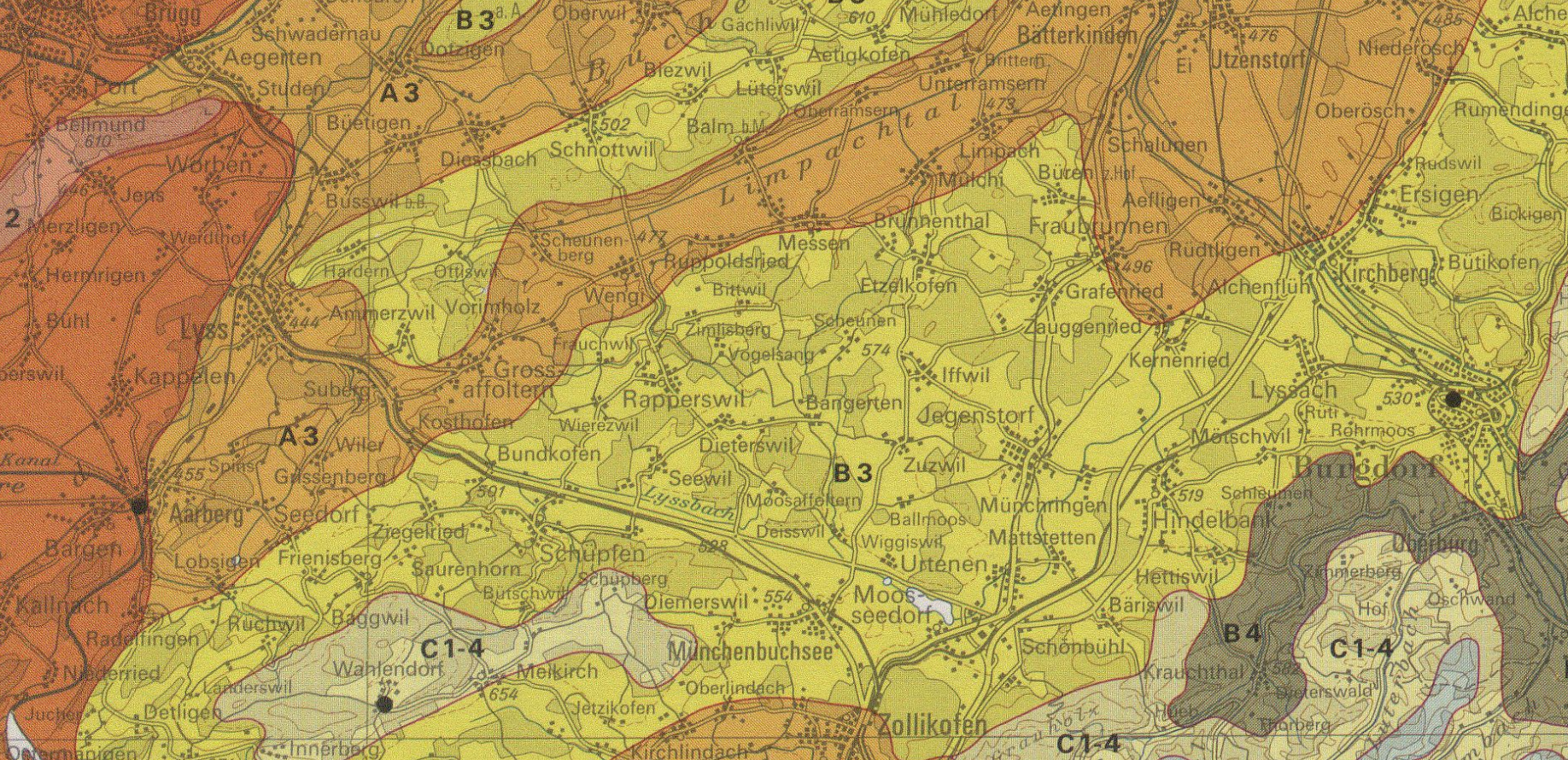

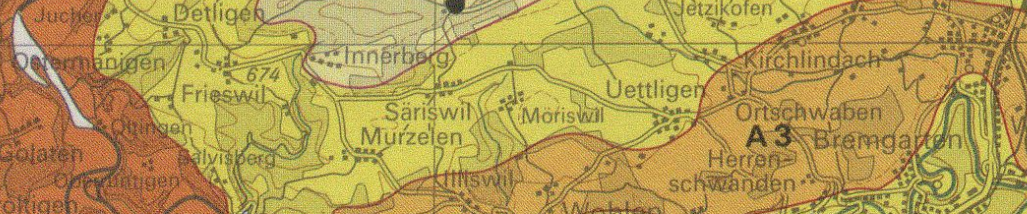

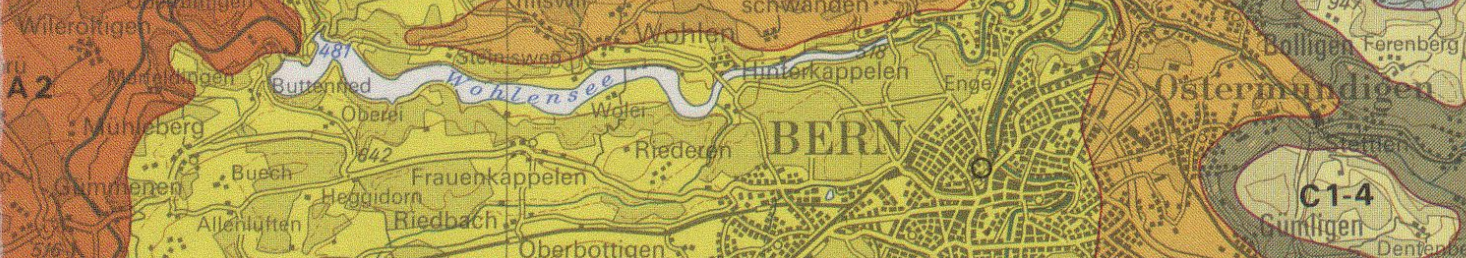

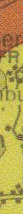

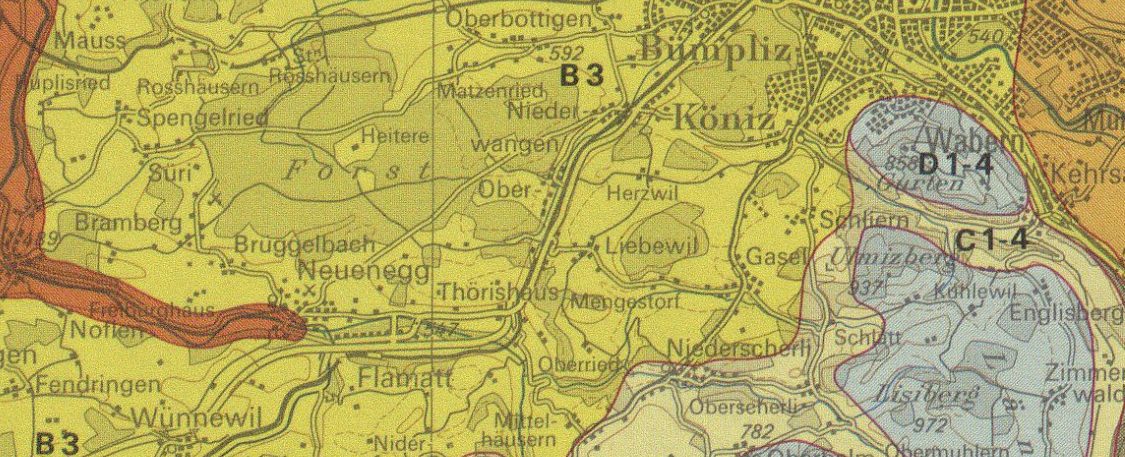

B̈3 
Abb.4: Ausschnitt aus der "Zusammenfassenden Legende" zur Klimaeignungskarte für die Landwirtschaft (siehe nebenstehend: Ausschnitt aus Blatt 1, 1:200'000); Bearbeitung: F.Jeanneret u. Ph. Vautier unter Mitwirkung von Dr. J. Caputa, Dr. R. Haeberli, Prof. Dr. B. Messerli u.a., Stand 1976; Herausgeber: Eidg. Justiz- u. Polizeidepartement, Der Delegierte für Raumplanung, u. Eidg. Volkswirtschaftsdepartement, Abt. für Landwirtschaft; Kartographie u. Druck: Eidg. Landestopographie, 3084 Wabern, 1977.

\begin{tabular}{|c|c|c|c|}
\hline Zone & Bezeichnung & Appellation & Denominazione \\
\hline$A_{1}$ & $\begin{array}{l}\text { Spezialkulturen } \\
\text { bevorzugut oder } \\
\text { begunstigt }\end{array}$ & $\begin{array}{l}\text { Cutures speciales } \\
\text { prifterentielles } \\
\text { ou favorisbes }\end{array}$ & $\begin{array}{l}\text { Collure speciali } \\
\text { preferenziali o } \\
\text { tavorizzate }\end{array}$ \\
\hline$A_{2}$ & $\begin{array}{l}\text { Acckerbou und } \\
\text { Sperailkulturen } \\
\text { begunstigt }\end{array}$ & $\begin{array}{l}\text { Grandes cultures et } \\
\text { culturess sptciales } \\
\text { tavorisees }\end{array}$ & $\begin{array}{l}\text { Grandi colture e } \\
\text { collure speciali } \\
\text { tavorizzate }\end{array}$ \\
\hline$A_{3}$ & $\begin{array}{l}\text { Ackerbau und } \\
\text { Funerbau } \\
\text { begunstigt }\end{array}$ & $\begin{array}{l}\text { Grandes cultures et } \\
\text { cultures lourragetres } \\
\text { lavonsees }\end{array}$ & $\begin{array}{l}\text { Grandi colture e } \\
\text { colture lotagogere } \\
\text { lavorizzate }\end{array}$ \\
\hline$A_{4}$ & $\begin{array}{l}\text { Furterbau } \\
\text { begunstigl }\end{array}$ & $\begin{array}{l}\text { Cultures fourragtres } \\
\text { favonisees }\end{array}$ & $\begin{array}{l}\text { Colture forroggere } \\
\text { Iavorizate }\end{array}$ \\
\hline$A_{5}$ & $\begin{array}{l}\text { Davergrunland } \\
\text { bevorzugt oder } \\
\text { begunstigt }\end{array}$ & $\begin{array}{l}\text { Herbages } \\
\text { permanents } \\
\text { pretérentiels } \\
\text { ou favorists }\end{array}$ & $\begin{array}{l}\text { Erbai permanenti } \\
\text { profeterenziali } \\
\text { ofevorizate }\end{array}$ \\
\hline$A_{B}$ & $\begin{array}{l}\text { Spezialikulturen } \\
\text { sentr begunstegt } \\
\text { Ackerbau } \\
\text { Wiessiand }\end{array}$ & $\begin{array}{l}\text { Cultures spbciales } \\
\text { utes favoristes } \\
\text { grandes cultures } \\
\text { herbages }\end{array}$ & $\begin{array}{l}\text { Colture speciali } \\
\text { tavorizazet } \\
\text { Grandicitione } \\
\text { Erbail }\end{array}$ \\
\hline $\mathrm{B}_{1}$ & $\begin{array}{l}\text { Ackerbau und } \\
\text { Spezialikulturen }\end{array}$ & $\begin{array}{l}\text { Grandes cultures ot } \\
\text { cultures sptciales }\end{array}$ & $\begin{array}{l}\text { Grandi colture e } \\
\text { colture speciali }\end{array}$ \\
\hline $\mathrm{B}_{2}$ & $\begin{array}{l}\text { Ackerbas und } \\
\text { funterbau }\end{array}$ & $\begin{array}{l}\text { Grandes cultures ot } \\
\text { cultures tourragtres }\end{array}$ & $\begin{array}{l}\text { Grandi colture e } \\
\text { colture loragoere }\end{array}$ \\
\hline $\mathrm{B}_{3}$ & $\begin{array}{l}\text { Funterbau und } \\
\text { Ackerbau }\end{array}$ & $\begin{array}{l}\text { Cultures lourragdres } \\
\text { et grandes cultures }\end{array}$ & $\begin{array}{l}\text { Colture loraggere } \\
\text { - grandi colture }\end{array}$ \\
\hline $\mathbf{B}_{\mathbf{4}}$ & Futrerbav & Cultures fourragatres & Colture foraggere \\
\hline$B_{5}$ & Davergrunland & $\begin{array}{l}\text { Herbages } \\
\text { permenents }\end{array}$ & Erbai permanenti \\
\hline $\mathrm{B}_{6}$ & $\begin{array}{l}\text { Dasuergiunland und } \\
\text { Spezialkulturen }\end{array}$ & $\begin{array}{l}\text { Herboges } \\
\text { permanents et } \\
\text { cultures speciales }\end{array}$ & $\begin{array}{l}\text { Erbal permanenti } \\
\text { e coiture specisti }\end{array}$ \\
\hline$C_{1-4}$ & $\begin{array}{l}\text { Furterbau und } \\
\text { Ackerbau. } \\
\text { mit Einsçrirankungen }\end{array}$ & $\begin{array}{l}\text { Cultures fourragetes } \\
\text { ol grandes cultures } \\
\text { avec restrictions }\end{array}$ & $\begin{array}{l}\text { Colture toreogere } \\
\text { e grandicicolture } \\
\text { con restrizione }\end{array}$ \\
\hline $\mathrm{C}_{5-6}$ & $\begin{array}{l}\text { Davergounland. } \\
\text { mit Einschrankungen }\end{array}$ & $\begin{array}{l}\text { Herboges } \\
\text { permanents } \\
\text { sevec contraintes }\end{array}$ & $\begin{array}{l}\text { Erbai permenenti } \\
\text { con rostrizioni }\end{array}$ \\
\hline$D_{1-4}$ & $\begin{array}{l}\text { Dauergiunland und } \\
\text { Ackerkulturen. } \\
\text { mit Einschrankingungen }\end{array}$ & $\begin{array}{l}\text { Herbeges } \\
\text { permenents } \\
\text { et cultives } \\
\text { avec testrictions }\end{array}$ & $\begin{array}{l}\text { Erbal permanenti } \\
\text { icolture con } \\
\text { restrizioni }\end{array}$ \\
\hline$D_{5-6}$ & $\begin{array}{l}\text { Oavergrunland. } \\
\text { mit starken } \\
\text { Einschrankungen }\end{array}$ & $\begin{array}{l}\text { Herboges } \\
\text { permonents } \\
\text { avec lories } \\
\text { contraintes }\end{array}$ & $\begin{array}{l}\text { Erboi permonenti } \\
\text { con torti } \\
\text { restrizioni }\end{array}$ \\
\hline$E_{1-3}$ & $\begin{array}{l}\text { Davuergrunlend } \\
\text { und einige } \\
\text { Ackerkulturen }\end{array}$ & $\begin{array}{l}\text { Herboges } \\
\text { permaneonts of } \\
\text { queiques cultires }\end{array}$ & $\begin{array}{l}\text { Erbai permanenti } \\
\text { oo alcune colture }\end{array}$ \\
\hline$E_{4-B}$ & $\begin{array}{l}\text { Davergrunland } \\
\text { bevorzugugt. } \\
\text { mit sterken } \\
\text { Einschrentungen } \\
\end{array}$ & $\begin{array}{l}\text { Herbegess naturels } \\
\text { pertererentiels avece } \\
\text { fortes contraintes }\end{array}$ & $\begin{array}{l}\text { Erbai naturali } \\
\text { prefoterenziali } \\
\text { con lorti } \\
\text { cestrizion } \\
\end{array}$ \\
\hline $\mathbf{F}$ & $\begin{array}{l}\text { Woiden und } \\
\text { Wiesen }\end{array}$ & $\begin{array}{l}\text { Paturages ot } \\
\text { pratries }\end{array}$ & Pascoli e preti \\
\hline G & Alpweiden & Alpages & Alpegoi \\
\hline & $\begin{array}{l}\text { Ausserhalb } \\
\text { Klassierung }\end{array}$ & Hors classement & Non classiticato \\
\hline
\end{tabular}

Niederschlagshaushalt auf der Alpensüdseite dafür verantwortlich ist, dass die Produktionsbedingungen trotz langer Vegetationsperiode und günstigen thermischen Voraussetzungen nicht für alle Kulturen ideal sind. Ebenso lässt sich demonstrieren, dass auch die Umgebung von Basel infolge gelegentlicher Trockenheit für Futterbau keine Höchsterträge erwarten lässt.

Die Einteilung in kombinierte Eignungskategorien zeigt deutlich, dass die einzelnen Kulturen spezifische Ansprüche an das Klima stellen. Das heisst, dass die einzelnen Zonen sich in ihrer Klimaeignung für die verschiedenen Kulturen recht deutlich unterscheiden.

\section{Anwendungen und Ausblick}

Die Klimaeignungskarten für die Landwirtschaft wurden insbesonders im Hinblick auf Anwendungen im Bereich der Raumplanung erstellt. Sie werden in den vorliegenden Massstäben vor allem für die Planung auf nationaler, kantonaler und regionaler Ebene geeignet sein. Vielleicht vermitteln diese Untersuchungen aber auch Informationen, die für den Agronomen, den $\mathrm{Kl}$ imatologen und den Geographen dienlich sind. Eine weitere Anwendung werden die Karten als Beilage zu einer Neuauflage der "Eidgenössischen Anleitung für die Schätzung landwirtschaftlicher Heimwesen und Liegenschaften" (1978) erfahren.

Ganz sicher offenbahren die Karten die Lage der begünstigten Landwirtschaftsgebiete in der Schweiz. Untersuchungen, wie diejenige von HAEBERLI (1975), zeigen, dass aber gerade in diesen Gegenden am meisten Kulturland verloren geht, wurden doch in den Jahren 1942-1967 100'000 ha oder fast $8 \%$ des Kulturlandes der landwirtschaftlichen Nutzung entzogen, und dies zu 78\% im Talgebiet.

Eine Verbesserung der vorliegenden $\mathrm{Kl}$ imaeignungskarte für die Landwirtschaft wird möglich, wenn :

- die agroklimatischen Schwellenwerte für kürzere Perioden zur Verfügung stehen (10 oder 5 Tage),

- die meteorologischen Beobachtungen eines grossen Teils des Stationsnetzes als Tageswerte abgelocht worden sind,

- das System von Perioden und Schwellenwerten auf weitere Kulturen ausgedehnt worden ist,

- agroklimatische Schwellenwerte auch für weitere meteorologische Elemente (Sonnenstrahlung, Bewölkung, Luftfeuchtigkeit usw.) vorliegen,

- Häufigkeit und Verbreitung meteorologischer Extremereignisse berücksichtigt werden können.

Schliesslich wird für die Raumplanung die Kombination der $\mathrm{Kl}$ imaeignung mit andern Elementen wesentlich sein, insbesonders der von FREY et alii (1977) erarbeiteten Bodeneignung, damit die Eignung für die Landwirtschaft generell für das ganze Land kartiert werden kann. Erst die Synthese aller wesentlicher Faktoren wird es der Raumplanung ermöglichen, die notwendigen Ents cheide auf fundierte Grundlagen zu stützen. 


\section{Bibliographie}

FREY,E. et alii, in Vorbereitung: Bodeneignungskarte der Schweiz (Massstab 1:200'000) = Carte des aptitudes des sols de la Suisse. Grundlagen für die Raumlpanung. Der Delegierte für Raumplanung, Abteilung für Landwirtschaft $u$. Oberforstinspektorat, Bern: Text +8 Karten.

HAEBERLI,R., 1971: Carte du potentiel naturel des surfaces agricoles du canton de Vaud. Cahier de l'aménagement régional 13, OCVU: $35 \mathrm{~S}$. +1 Karte.

HAEBERLI,R., 1975: Verlust an landwirtschaftlicher Kulturfläche. Informationshefte des Delegierten für Raumplanung 1975(2): $15 \mathrm{~S}$.

IMHOF,E., ab 1965 (Hrsg.): Atlas der Schweiz. Eidg.Landestopographie, Wabern-Bern.

IMHOF,E., 1972: Thematische Kartographie. Lehrbuch der Allg.Geographie X, Berlin: $360 \mathrm{~S} .+$ Beilagen.

JEANNERET,F., 1975a: Die Verteilung der Spätfrostschäden in der Schweiz im Frühling 1974. Schweiz. landwirtschaftl. Forschung 15(1): 1-14 + Karte - Informationen u. Beiträge zur Klimaforschung, Bern 13: 15 S.+ Karte.

JEANNERET,F., 1975b: Grundlagen zum Klima der Schweiz: Klimatolog.Bibliographie 1921-1973. Klimatologie der Schweiz N, Beiheft zu den Annalen der Schweiz.Meteorolog.Zentralanstalt 1974 - Geographica Bernensia G3: 124 S. + 4 Karten.

JEANNERET,F.; VAUTIER,Ph., 1977a: Kartierung der $K l$ imaeignung für die Landwirtschaft in der Schweiz = Levé cartographique des aptitudes climatiques pour l'agriculture en Suisse. Beiheft 4 zum Jahrbuch der Geogr. Gesell schaft von Bern 1977.
JEANNERET,F.; VAUTIER,Ph.,1977b: KI imaeignungskarten für die Landwirtschaft in der Schweiz = Cartes climatiques pour l'agriculture en Suisse. Grundlagen für die Raumplanung, Der Delegierte für Raumplanung u.Abteilung für Landwirtschaft, Bern: $57 \mathrm{~S} .+6$ Karten.

LEUZINGER,H. ,1978: Grundlagenkarten für die Raumplanung. Geographica Helvetica 1/1978, S. $17-20$.

MAEDER,F., 1970: Hinweise u. Quellenangaben zu den Klimaeignungskarten. Im Auftrag des Instituts für Orts-, Regional-u. Landesplanung der ETH-Z: 23 S. + 2 Karten.

PRIMAULT,B.,1968: Der Einfluss des Klimas auf die Entwicklung einer Kultur. Dargestellt am Beispiel von vier Körnermaissorten. Die Grüne 1968 (29): 1065-1068

PRIMAULT,B., 1972a: Ausreifwahrscheinlichkeit von Körnermais bis 15. Oktober. Agrarmeteorologische Blätter der Eidg. Forschungsanstalt für Betriebswirtschaft u. Landtechnik Tänikon : 2 Karten.

PRIMAULT,B., 1972b: Etude mésoclimatique du Canton de Vaud. Cahier de 1 'amënagement régional 14 OCVU: $186 \mathrm{~S}$.

SCHREIBER,K.F.; KUHN,N.; HUG,C.; HAEBERLI,R.; SCHREIBER,C., 1977: Wärmegl iederung der Schweiz = Niveaux thermiques de la Suisse. Grundlagen für die Raumplanung, Bern: Text +5 Karten.

SCHWEIZERISCHE METEOROLOGISCHE ZENTRALANSTALT, ab 1959: Klimatologie der Schweiz. Beihefte zu den Annalen der MZA Zürich.

UTTINGER,H., 1949: Die Niederschlagsmengen in der Schweiz 1901 - 1940. Führer durch die schweizerische Wasser- u. Energiewirtschaft 2 (3): 27 S. + 1 Karte.

\section{Literaturbesprechung}

BLESSING Heinrich Michael, Karstmorphologische Studien in den Berner Alpen, Tübinger geogr. Studien, Heft 65,77 S., 3 Karten, 8 Abb., 15 Photos, Selbstverlag des Geogr. Inst. d.Univ., Tübingen 1976, DM 30.--.

Der Autor hat sich zum Ziele gesetzt, drei alpine Karstgebiete verschiedener Höhe im weiteren Umkreise der Berner Alpen zu beschreiben, zu vergleichen und ihre Entstehung zu deuten. Es sind dies das Wildhorngebiet $(2300 \mathrm{~m}-2700 \mathrm{~m}$ ïM), die Faulhorngruppe (1400 - 2400m üM) und das Brüniggebiet (unter $1400 \mathrm{~m}$ ). Ein besonderes Gewicht wurde auf die Faktoren der Struktur, der Altformen, der pleistozänen Vergletscherung, der Verkar- stung, der Petrovarianz und der Klimavarianz für die Entstehung und Verteilung der Formen gelegt. Der grosse Umfang des Arbeitsgebietes, gemessen an der zur Verfügung stehenden Zeit, hat es offensichtlich nicht gestattet, alle bei diesen Untersuchungen auftretenden Probleme weiter zu verfolgen und die Beweise dafür zu erarbeiten. Es ist schade, dass die Arbeiten über die Morphogenese der Schweizer Alpen nicht für die Datierung der Altformen herangezogen wurden. Der Autor hat aber mit dieser Arbeit eine Grundlage voller Anregungen geschaffen, die aufgegriffen und weiter verfolgt werden sollten. GH $1 / 1978$

A. Bögli 